\title{
Quantum extreme black holes at finite temperature and exactly solvable models of $2 \mathrm{~d}$ dilaton gravity
}

\author{
O. B. Zaslavskii \\ Department of Physics, Kharkov Karazin's National University, Svobody Sq.4, Kharkov \\ 6107r, Ukraine \\ E-mail: aptm@kharkov.ua

\begin{abstract}
It is argued that in certain $2 \mathrm{~d}$ dilaton gravity theories there exist selfconsistent solutions of field equations with quantum terms which describe extreme black holes at nonzero temperature. The curvature remains finite on the horizon due to cancelation of thermal divergencies in the stress-energy tensor against divergencies in the classical part of field equations. The extreme black hole solutions under discussion are due to quantum effects only and do not have classical counterparts.
\end{abstract}

PACS numbers: 04.60Kz, 04.70.Dy

\section{INTRODUCTION}

In [1] it was argued that thermodynamic reasonings allow one to ascribe nonzero temperature $T$ to extreme black holes since the Euclidean geometry remains regular irrespectively of the value of $T$. As a result, it was conjectured that an extreme black hole may be in thermal equilibrium with ambient radiation at any temperature. This conclusion was criticized in [2] where it was pointed out that the prescription of [1] is suitable only in the zero-loop approximation and does not survive at quantum level since the allowance for quantum backreaction leads to thermal divergencies in the stress-energy tensor that seems to destroy a regular horizon completely, so the demand of regularity of this tensor on the horizon seems to enforce the choice $T=0$ for extreme black holes unambiguously.

The aim of the present paper is to show that in dilaton gravity there exists possibility to combine a finite curvature at the horizon with divergencies of the stress-energy tensor $T_{\mu}^{\nu}$. 
As a result, extreme black holes with $T \neq 0$ and finite curvature on the horizon may exist. Namely, the above mentioned divergencies may under certain conditions be compensated by the corresponding divergencies in the classical part of field equations due to derivatives of the dilaton field. We stress that the geometries discussed below are self-consistent solutions of field equations with backreaction of quantum fields taken into account. In spite of the geometry itself turns out to be regular in the sense that the curvature measured from outside is finite at the horizon, the solution as the whole which includes, apart from the metric, the dilaton field as well, is singular. (The similar result was obtained quite recently for nonextreme black holes [0]).

It was observed earlier that "standard" extreme black holes with $T=0$ exhibit some weak divergencies at the horizon [4]. The phenomenon we are discussing is qualitatively different. First, these divergencies are inherent in our case to the components $T_{\mu}^{\nu}$ themselves, whereas in 四 $T_{\mu}^{\nu}$ were assumed to be finite and divergencies revealed themselves in the energy measured by a free falling observer which is proportional to the ratio $\left(T_{1}^{1}-T_{0}^{0}\right) / g_{00}\left(x^{0}\right.$ and $x^{1}$ are a temporal and spatial coordinates in the Schwarzschild gauge). Second, divergencies considered in [四] arise for a generic extreme black hole with $T=0$, whereas in our case they are due to $T \neq 0$ entirely. Third, backreaction of quantum field produces a singularity at the horizon in [⿶凵

It is worth noting that in quantum domain the issue of existence of extreme black holes is non-trivial by itself. In Ref. [0] it was argued that the existence of quantum extreme black holes is inconsistent with field equations at all. However, this conclusion was derived for the particular class of CGHS-like models [6], so its region of validity is very limited, as, in fact, the authors themselves note at the end of their paper. In the present paper we show that for a certain class of dilaton gravity theories (i) extreme black holes with quantum corrections taken into account do exist; (ii) the temperature of quantum fields in their background may be nonzero. We exploit the approach which was elaborated earlier in Refs. [7], [8], where it was applied to nonextreme black holes and semi-infinite throats. 


\section{GENERAL STRUCTURE OF SOLUTIONS}

Consider the action of the dilaton gravity

$$
I=I_{0}+I_{P L}
$$

where

$$
I_{0}=\frac{1}{2 \pi} \int_{M} d^{2} x \sqrt{-g}\left[F(\phi) R+V(\phi)(\nabla \phi)^{2}+U(\phi)\right]
$$

and the Polyakov-Liouville action [9] incorporating effects of Hawking radiation and its backreaction on the black hole metric can be written as

$$
I_{P L}=-\frac{\kappa}{2 \pi} \int_{M} d^{2} x \sqrt{-g}\left[\frac{(\nabla \psi)^{2}}{2}+\psi R\right]
$$

The function $\psi$ obeys the equation

$$
\square \psi=R,
$$

where $\square=\nabla_{\mu} \nabla^{\mu}, \kappa=N / 24$ is the quantum coupling parameter, $N$ is number of scalar massless fields, $R$ is a Riemann curvature. We omit the boundary terms in the action as we are interested only in field equations and their solutions.

A generic quantum dilaton-gravity system is not integrable. However, if the action coefficients obey the relationship

$$
V=\omega\left(u-\frac{\kappa \omega}{2}\right)
$$

where $\omega=U^{\prime} / U$, the system becomes exactly solvable [10], [7]. Then static solution are found explicitly and even for a finite arbitrary temperature $T$ of quantum fields measured at infinity [8]:

$$
\begin{aligned}
d s^{2} & =g\left(-d t^{2}+d \sigma^{2}\right), g=\exp \left(-\psi_{0}+2 y\right), y=\lambda \sigma, \psi_{0}=\int \omega d \phi=\ln U(\phi)+\text { const. } \\
F^{(0)}(\phi) & =f(y) \equiv e^{2 y}-B y+C, B=\kappa\left(1-T^{2} / T_{0}^{2}\right), T_{0}=\lambda / 2 \pi,
\end{aligned}
$$


and the auxiliary function $\psi=\psi_{0}+\gamma \sigma, \gamma=2 \lambda\left(T / T_{0}-1\right)$. Here $F^{(0)}=F-\kappa \psi_{0}, y=\lambda \sigma$, $\lambda=\sqrt{U} / 2$. At right infinity, where spacetime is supposed to be flat, the stress-energy tensor has the form

$$
T_{\mu}^{\nu(P L)}=\frac{\pi N}{6} T^{2}(1,-1)
$$

The coordinate $\sigma$ is related to the Schwarzschild one $x$, where

$$
d s^{2}=-d t^{2} g+g^{-1} d x^{2}
$$

by the formula $x=\int g d \sigma$. We assume that the function $\omega(\phi)$ changes its sign nowhere, so in what follows we may safely use the quantity $\psi_{0}$ instead of $\phi$ (in fact, this can be considered as reparametrization of the dilaton).

The solutions (6) include different types of object - nonextreme black holes, "semi-infinite throats" and soliton-like solutions, depending on the boundary conditions imposed on the function $\psi_{0}$ on the horizon [8]. Now we want to elucidate whether the models (5) include also extreme black holes with a finite curvature at the horizon and under what conditions. Near a horizon the metric of a typical extreme black hole must have the standard form (subscript " $\mathrm{h}$ " indicates that a quantity is calculated at the horizon)

$$
g \approx g_{0}\left(x-x_{h}\right)^{2} \approx \frac{g_{0}}{y^{2}}
$$

or, equivalently,

$$
\psi_{0} \approx 2 y+\ln y^{2}
$$

At the right infinity the function $\psi_{0}$ must obey the relation

$$
\psi_{0} \approx 2 y
$$

that a spacetime have the Minkowski form and, thus, $\psi_{0} \rightarrow+\infty$ in accordance with general properties indicated in [11].

Provided eqs. (9) and (10) are satisfied, the Hawking temperature 


$$
T_{H}=\frac{1}{4 \pi}\left(\frac{d g}{d x}\right)_{h}=\lim _{y \rightarrow-\infty} \frac{\lambda d g}{4 \pi g d y}=\frac{\lambda}{2 \pi} \lim _{y \rightarrow-\infty}\left(1-\frac{1}{2} \frac{d \psi_{0}}{d y}\right)=0
$$

as it should be for an extreme black hole, the Riemann curvature $R=-\lambda^{2} g^{-1} \frac{d^{2}}{d y^{2}} \ln g \approx$ $-2 g_{0}^{-1} \lambda^{2}$ is finite. As near the horizon $y \rightarrow-\infty, f(y) \approx-B y$, we obtain that the function

$$
F^{(0)} \approx-\frac{B}{2}\left(\psi_{0}-\ln \frac{\psi_{0}^{2}}{4}\right)
$$

at $\psi \rightarrow-\infty$.

If eq. (13) is satisfied, the function $\psi_{0}(\phi)$ which is the solution of (6) has the desirable asymptotic (10) and, therefore, the metric function behaves according to (9). To achieve the behavior (11) at infinity, it is sufficient to enforce the condition $F^{(0)} \approx e^{\psi_{0}}$ at $\psi \rightarrow+\infty$.

As we are looking for solutions of field equations which are regular in the region between the horizon and infinity, we must exclude a possible singularity of curvature. Since $R=$ $-\lambda^{2} g^{-1} \frac{d^{2} \psi_{0}}{d y^{2}}$ and $\frac{d \psi_{0}}{d y}=\frac{f^{\prime}(y)}{F^{\prime}\left(\psi_{0}\right)}$ (prime denotes derivative with respect to a corresponding argument), the dangerous point is $\psi_{0}^{*}$ where $F^{(0) \prime}\left(\psi_{0}^{*}\right)=0$ and $\frac{d \psi_{0}}{d y}$ may diverges. Let $B>0$. Then $\frac{d \psi_{0}}{d y}$ is finite in the corresponding point $y^{*}$, provided $f^{\prime}\left(y^{*}\right)=0$. It is achieved by the choice $C=F^{(0)}\left(\psi_{0}^{*}\right)+\frac{B}{2}\left(\ln \frac{B}{2}-1\right) \equiv C^{*}$. If $B<0$, the function $F^{(0)}\left(\psi_{0}\right)$ must be monotonic to ensure the absence of singularities. For $B=0$, as we will see below, extreme black holes do not exist.

Let us consider an example for which all above-mentioned conditions are satisfied:

$$
F^{(0)}=e^{2 \gamma}-B \gamma+C^{*},
$$

where $\gamma=\gamma\left(\psi_{0}\right)$. Then eq. (6) has the obvious solution: $y=\gamma\left(\psi_{0}\right)$. If we choose the function $\gamma$ in such a way that the solution of this equation $\psi_{0}(y)$ has the needed asymptotics (10), we obtain an extreme black hole in accordance with (9). It is also clear that $F^{(0) \prime}=0$ in the same point where $f^{\prime}(y)=0$, so the condition of absence of singularities is satisfied.

Let us look now at the behavior of the components of the stress-energy tensor of quantum field near the horizon. For definiteness, let us choose the $T_{1}^{1}$ component. It can be written in the Schwarzschild gauge as (see, for example, [2]; it is assumed that the field is conformally invariant, our definition of $T_{\mu}^{\nu(P L)}$ differs by sign from the quantum stresses discussed in [2]) 


$$
T_{1}^{1(P L)}=\frac{\pi}{6 g}\left[T^{2}-\left(\frac{g^{\prime}}{4 \pi}\right)^{2}\right] .
$$

Since in our case $T \neq T_{H}$, the expression in square brackets remains nonzero at the horizon, $T_{1}^{1} \sim g^{-1} \sim\left(x-x_{h}\right)^{-2} \sim y^{2}$, so stresses diverge strongly.

\section{PROPERTIES OF SOLUTIONS}

It was observed in [7] that for black holes described exact solutions (6) the Hawking temperature $T_{H}=\lambda / 2 \pi$ is nonzero constant irrespectively of the particular kind of the model that generalizes the earlier observation [12] for the RST model [13]. Therefore, it would seem that black holes entering the class of solutions under discussion may be nonextreme only. The reason why, along with nonextreme holes, the class of solutions contains, nevertheless, extreme ones too, can be explained in terms of the function $\psi$. It was assumed in [12] and [7] that this quantity is finite on the horizon to ensure the finiteness of the Riemann curvature.

Then the derivative $\frac{d \psi}{d y} \rightarrow 0$ at the horizon, where $y \rightarrow-\infty$, and $T_{H}=\lambda / 2 \pi$ in accordance with (12). However, we saw that even notwithstanding $\psi$ diverges on the horizon, we can find the solutions with a finite $R$ on the horizon, provided the function in question has the asymptotic (10). Thus, we gain qualitatively new types of solutions due to relaxing the condition of the regularity of $\psi$ on the horizon.

It is worth paying attention to two nontrivial features of solutions under discussion: (i) the character of solutions in the classical $(\kappa=0)$ and quantum $(\kappa \neq 0)$ cases is qualitatively different, (ii) extreme black holes with a finite curvature at the horizon are possible even if $T \neq T_{H}=0$. First, let us consider the point (i). Even if $T=0$, in the quantum case the coefficient $B \neq 0$ and $f \rightarrow \infty$ at the horizon, whereas in the classical case $(B=0)$ $f \rightarrow C=$ const. Therefore, in the classical case the value of the coupling coefficient $F$ is finite on the horizon: according to (6),$F_{h}=F_{h}^{(0)}=C$. As a result, in the vicinity of the horizon $\psi_{0}=\psi_{0 h}$ plus exponentially small corrections, so $\psi_{0}(y)$ is regular and a nonextreme black hole becomes nonextreme, as explained in the precedent paragraph. On the other hand, $F^{(0)}$ must diverge at the horizon in the quantum case. Thus, we arrive at a rather 
unexpected conclusion: for exactly solvable models of dilaton gravity (5) extreme black holes are due to quantum effects only and disappear in the classical case $\kappa=0$. This fact is insensitive to the value of temperature, so classical extreme black holes of the given type are absent even if $T=0$. For the same reasons quantum extreme black hole are absent in the exceptional case $T=T_{0}$, when $B=0$.

It was observed in [5] that for a rather wide class of Lagrangians the typical situation is such that classical extreme black holes may exist, whereas quantum correction to the action destroy the character of the solution completely and do not allow the existence of extreme black holes. In our case, however, the situation is completely opposite: extreme black holes are possible in the quantum (but not classical) case. It goes without saying that our result does not contradict the existence of extreme black holes in the classical dilaton gravity theories in general but concerns only the special class of Lagrangians (5) and their classical limit. (It is worth recalling that the condition (5) was imposed to ensure the solvability of the quantum theory, whereas in the classical case every dilaton-gravity model is integrable, so there is no need in supplementary conditions like (5)). The results of either [5] or the present paper show clearly that quantum effects not only may lead to quantum corrections of the classical metric but radically change the character of the geometry and topology.

The most interesting feature of solutions obtained in the present paper is the possibility to have black holes at $T \neq T_{H}=0$. In the previous paper [3] we showed that nonextreme black holes with $T \neq T_{H}$ may exist. The reason in both cases is the same and I repeat it shortly. The usual argument to reject the possibility $T \neq T_{H}$ relies on two facts: 1) this inequality makes the behavior of the stress-energy tensor of quantum fields singular in the vicinity of the horizon, 2) in turn, such a behavior of the stress-energy tensor is implied to inevitably destroy a regular horizon by strong backreaction. Meanwhile, a new subtlety appears for dilaton gravity as compared to the usual case. As, in addition to a metric and quantum fields, there is one more object - dilaton, there exists the possibility that divergencies in the stress-energy tensor are compensated completely by gradients of a dilaton field to give a metric regular at the horizon (at least, from outside). And for some 
models, provided the conditions describe above are fulfilled, this situation is indeed realized. Thus, dilaton gravity shares the point 1) with general relativity but the condition 2) may break down.

It is worthwhile to note that, as follows from (6), the temperature which determines the asymptotic value of the energy density at infinity, is the function of the given coefficient $B$ which enters the form of the action coefficient $F^{(0)}(\psi): T=T_{0} \sqrt{1-B / \kappa}$, so the solution under discussion has sense for $B \leq \kappa$ only. In particular, for "standard" extreme holes with $T=0$ the coefficient $B=\kappa \neq 0$. It follows from (5), (10), (10), that near the horizon (i.e. in the limit $y \rightarrow-\infty)$ the coefficient $V$ in the action (国) behaves like $V \approx \frac{\omega^{2}}{2}(\kappa-B)$, so the condition $B \leq \kappa$ ensures the right sign of the term with $(\nabla \phi)^{2}$.

The fact that for a given $B$ we obtain the fixed value of the temperature is contrasted with that for nonextreme black holes with $T \neq T_{H}$ where the temperature is not fixed by the form of the action but takes its value within the whole range [3]. This can be explained as follows. In the extreme case we must achieve $T_{H}=0$ that entails fine tuning in the asymptotic behavior of $\psi_{0}(y)$ that forces us to choose the coefficient at the linear part of $F^{(0)}$ equal to $B$ exactly. Meanwhile, in the nonextreme case, where the value of $T_{H}$ is not fixed beforehand, the corresponding coefficient is bounded only by two conditions which guarantee the existence of the horizon and the finiteness of the curvature. These conditions result in two inequalities, so the constraint is less restrictive (see [3] for details).

It is worth recalling that Trivedi has found nonanalytical behavior of the metric of extremal black holes near the horizon: $g \approx \alpha_{1}\left(x-x_{h}\right)^{2}+\alpha_{2}\left(x-x_{h}\right)^{3+\delta}$ (see eq. (27) of «). In our case corrections to the leading term of (9) also may be nonanalytical, depending on the properties of the function $F^{(0)}\left(\psi_{0}\right)$ (for the example (14) all is determined by the choice of the function $\left.\gamma\left(\psi_{0}\right)\right)$. Moreover, it is readily seen that in our case the nonanaliticity may reveal itself in the leading term of $g$. Indeed, let the function $g$ have the asymptotic

$$
g \approx g_{0}\left(x-x_{h}\right)^{\delta}
$$

with $\delta>2$ that is compatible with the extremality condition $T_{H}=0$. This case can be 
handled in the same manner as for $\delta=2$. From (6) and (8) it follows that now we must have near the horizon the asymptotics

$$
\psi_{0} \approx 2 y+\alpha \ln (-y),
$$

with $\alpha=\delta /(\delta-1)$ instead of (10). One more case arises when $g \approx g_{0} e^{-x}$, so $y \sim e^{x}$, the horizon lies at $x=\infty$. Then we obtain the same formula (17) with $\alpha=1$.

From the physical viewpoint, the model considered in [4] represents charged black holes in the dilaton theory motivated by the reduction from four dimensions. The corresponding model is not exactly solvable either due to the presence of the electromagnetic field or due to the form of the action coefficients which do not fall into the class of exactly solvable models [7], [10]. On the other hand, our model is exactly solvable and deals with uncharged black holes. Their extremality is due to quantum effects entirely.

The relevant physical object in dilaton gravity includes not only a metric but both the metric and dilaton field. In the situations analyzed above the coupling $F^{(0)}$ between curvature and dilaton describe inevitably diverges at the horizon as seen from eq. (6) in the limit $y \rightarrow-\infty$ corresponding to the horizon. Therefore, although the curvature is finite on the horizon, the solution as the whole exhibit singular behavior. Moreover, even the metric part of the solution can be called "regular" only in the very restricted sense: the region inside the horizon hardly has a physical meaning at all since an outer observer cannot penetrate it because of strong divergencies in the stress-energy tensor on the horizon surface, so the manifold is geodesically incomplete. All these features tell us that in fact we deal with the class of objects which occupies the intermediate positions between "standard" regular black holes and naked singularities. In the previous paper we described the nonextreme type of such objects, in the present paper we found its extreme version.

\section{SUMMARY}

We have proved that extreme black holes do exist in exactly solvable models of dilaton gravity with quantum corrections taken into account. It turned out that they are contained in 
general solutions of exactly solvable models. The existence of extreme black holes depends strongly on the behavior of the action coefficients near the horizon (see eq. (13)) and is insensitive to their concrete form far from it where it is only assumed that their dependence on the dilaton field ensures the absence of singularities.

We found solutions which shares features of both black holes and naked singularities and represent "singularities without singularities". We showed that quantum fields propagating in the background of extreme black holes may nonzero temperature at infinity. In fact, this means that such fields cannot be called Hawking radiation since the geometry does not enforce the value of the temperature. These properties of solutions with $T \neq T_{H}$ are the same as in the nonextreme case [3]. The qualitatively new feature inherent to extreme black holes consists in that the type of solutions we deal with is very sensitive to quantum effects: taking the classical limit destroys completely our solutions with $T \neq T_{H}=0$, so the solutions under discussion are due to quantum effects only. (In contrast to it, solutions describing nonextreme black holes with $T \neq T_{H}$ considered in [3] have sense in the classical limit.)

The separate issue which deserves separate consideration is what value of entropy should be ascribed to the objects we found.

I am grateful to Sergey Solodukhin for valuable comments.

[1] S.W. Hawking, G.T. Horowitz, S.F. Ross, Phys.Rev. D 51 (1995) 4302.

[2] D.J. Lorantz, W.A. Hiscock, P. R. Anderson, Phys.Rev. D 52 (1995) 4554.

[3] O. B. Zaslavskii, Two-dimensional dilaton gravity and spacetimes with finite curvature at the horizon away from the Hawking temperature, hep-th/9911077 (Phys. Rev. D, in press).

[4] S. Trivedi, Phys. Rev. D 47 (1993) 4233.

[5] G. Alejandro, F. D. Mazzitelli, and G. Núñez, Phys. Lett. B. 355 (1995) 92. 
[6] C. G. Callan, S. Giddings, J. A. Harvey, and A. Strominger, Phys. Rev. D 45 (1992) R1005.

[7] O. B. Zaslavskii, Phys. Rev. D 59 (1999) 084013.

[8] O. B. Zaslavskii, Phys. Lett. B Phys. Lett. B 459 (1999)105.

[9] A. M. Polyakov, Phys. Lett. B 103 (1981) 207.

[10] Y. Kazama, Y. Satoh, and A. Tsuichiya, Phys. Rev. D 51 (1995) 4265.

[11] S. N. Solodukhin, Phys. Rev. D 51 (1995) 609.

[12] S. N. Solodukhin, Phys. Rev. D 53 (1996) 824.

[13] J. G. Russo, L. Susskind, L. Thorlacius, Phys. Rev. D 46 (1992) 3444; Phys. Rev. D 47 (1992) 533. 